\title{
Clinical presentation of alcoholic liver disease and non-alcoholic fatty liver disease: spectrum and diagnosis
}

\author{
Praveen Sharma, Anil Arora \\ Department of Gastroenterology \& Hepatology, Sir Ganga Ram Hospital, New Delhi, India \\ Contributions: (I) Conception and design: All authors; (II) Administrative support: None; (III) Provision of study material or patients: None; (IV) \\ Collection and assembly of data: None; (V) Data analysis and interpretation: All authors; (VI) Manuscript writing: All authors; (VII) Final approval of \\ manuscript: All authors. \\ Correspondence to: Dr. Anil Arora, MD, DM. Professor, Department of Gastroenterology, Sir Ganga Ram Hospital, New Delhi, India. \\ Email: dranilarora50@gmail.com.
}

\begin{abstract}
Alcoholic liver disease (ALD) and non-alcoholic fatty liver disease (NAFLD) are commonest causes of chronic liver disease in developing as well as developed countries. Their incidence has increased due to widespread easy availability of alcohol and sedentary life style of people. NAFLD is a spectrum which includes fatty liver (NAFL) which is considered benign disease, steatohepatitis (NASH) which indicates ongoing injury to liver and cirrhosis of liver. Similarly, ALD spectrum comprises simple steatosis, alcoholic hepatitis, and cirrhosis and its complications. Most of the time there is significant overlap between these diseases and clinical presentation depends upon the stage of liver disease. Most of the NAFLD patients are asymptomatic and diagnosed to have fatty liver while undergoing routine health check up. ALD requires significant history of alcohol intake which is supportive by radiological and biochemical tests. In both NAFLD and ALD patients, liver enzymes are seldom raised beyond five times the upper limit of normal. Liver biopsy is required for diagnosis of NASH as it is a histological diagnosis and sometimes in alcoholic hepatitis for confirmation if diagnosis is in doubt. Non-invasive markers and prognostic scores have been developed for avoiding liver biopsy in assessment and treatment response of NASH and alcoholic hepatitis patients.
\end{abstract}

Keywords: Alcoholic liver disease (ALD); non-alcoholic fatty liver disease (NAFLD); clinical presentation; diagnosis; spectrum

Received: 29 July 2019; Accepted: 05 October 2019; Published: 05 April 2020.

doi: $10.21037 / \operatorname{tgh} .2019 .10 .02$

View this article at: http://dx.doi.org/10.21037/tgh.2019.10.02

Alcoholic (ALD) and non-alcoholic (NAFLD) liver disease are one of commonest etiologies of chronic liver disease all over the world $(1,2)$. Easy accessibility to food and sedentary life style has led to obesity and associated problems in many countries. Alcohol consumption has increased in all countries which have led to increased morbidity, hospital admission and mortality from ALD. NAFLD spectrum includes fatty liver (NAFL), steatohepatitis (NASH) and cirrhosis. Similarly, ALD spectrum includes simple steatosis, alcoholic hepatitis (AH), combination of $\mathrm{AH}$ and cirrhosis and cirrhosis. These pathologic processes often overlap in NAFL and ALD. This review highlights the clinical spectrum and diagnosis of ALD and NAFLD $(3,4)$.

\section{ALD}

The diagnosis of ALD is made based on reliable history of significant alcohol intake, clinical examination and laboratory features suggestive of significant alcohol intake. Definition of one standard alcoholic drink is any alcoholic beverage that contains $14 \mathrm{~g}$ of alcohol. In men consuming more than 4 standard drinks in any single day (or more than 14 drinks per week) is considered significant alcohol intake, similarly in women more than 3 standard drinks in any 
single day (or more than 7 drinks per week) is considered significant to cause alcohol-related problems (3).

However reliable history of significant alcohol intake is clinically challenging in many patients and making a diagnosis of ALD need assessment by screening tools and laboratory tests. Several screening tools are available that are validated in many studies and can be easily administered during a clinical visit are available to identify patients at risk for alcohol abuse. Off the many tools available alcohol use disorders identification test (AUDIT), AUDITconsumption (AUDIT-C) and single question screening tool are the common screening tools in primary care setting. AUDIT is the most widely studied for detecting alcohol use disorders by many physicians $(5,6)$.

No single laboratory or imaging study can confirm the diagnosis of ALD. A detailed history from relatives is crucial in suspecting liver disease due to alcohol. Furthermore, in the initial stages of disease most of the patients are completely asymptomatic, have no clinical signs of liver disease or early cirrhosis and these patients also have normal or slightly raised liver enzymes. Alcoholic patients may have co-existing risk factors for NAFLD such as obesity, diabetes and hepatitis $\mathrm{C}$ in patients with ALD. In these patients, significant amount of alcohol varies as even smaller amount of alcohol can cause significant liver injury $(7,8)$.

In outpatient or inpatient department a diagnosis of ALD should be suspected in patients with a significant alcohol abuse who present with abnormal serum transaminases, level of aspartate aminotransferase (AST) which is greater than that of alanine aminotransferase (ALT), clinical finding of hepatomegaly with signs of chronic liver disease, and radiological evidence of liver steatosis or fibrosis/cirrhosis. Liver biopsy if done shows macrovesicular steatosis, fibrosis or cirrhosis or combination of these findings (3).

\section{Liver enzymes in the diagnosis of ALD}

Liver enzymes are not very useful for the diagnosis of patients with ALD as liver enzymes may or may not be elevated and the elevation does not correlate with severity of disease. However, the pattern of elevation is helpful in making a diagnosis of liver injury due to alcohol as AST is typically two to three times greater than ALT in these patients. Majority of times the levels of AST and ALT will not increase 5 times the upper level of normal and in any patient if liver enzymes level are more than 8-10 times the upper level of normal a second diagnosis should be considered $(3,9)$. Another clue to diagnosis is elevated serum gamma-glutamyltranspeptidase (GGT) which is more than serum alkaline phosphatase level. If a patient has low serum albumin, low platelet and high prothrombin time then advanced fibrosis or cirrhosis need to be considered. However, it is pertinent to exclude other etiologies before making a definitive diagnosis of ALD, and relevant tests to exclude chronic viral hepatitis (hepatitis B and $\mathrm{C}$ ), autoimmune hepatitis, hemochromatosis, Wilson disease and drug related hepatotoxicity be done. When the diagnosis is unclear, a liver biopsy may be considered to exclude other causes of liver disease.

\section{Common bematological finding}

Common findings include low platelet count, anemia with high mean corpuscular volume (MCV), low lymphocyte with raised neutrophil count, elevated erythrocyte sedimentation rate and an elevated prothrombin time (international normalised ration, INR). Elevated INR with low albumin suggests advanced liver disease. Patients with ALD frequently have evidence of iron overload which is reflected by elevated serum iron indices (ferritin and transferrin saturation) (10). Many biochemical markers have been evaluated for chronic alcohol consumption in these patients and include serum GGT, AST/ALT ratio, $\mathrm{MCV}$ and carbohydrate-deficient transferrin (CDT). CDT combined with GGT has sensitivity of about $80-90 \%$ in identifying patients with alcohol abuse. However, CDT levels may be fallacious with increasing disease severity and active smoking (11). Newer biomarkers such as ethyl glucuronide (metabolites of alcohol) can identify alcohol use up to 3-4 days after the last alcohol drink (12). However, till date no single biomarker has both high sensitivity and specificity for detecting chronic alcohol abuse. But a combination of markers may provide better diagnostic yield in the diagnosis for example, CDT in combination with other biomarker like GGT and/or MCV, improves sensitivity significantly $(13,14)$.

\section{Role of liver biopsy in diagnosis of spectrum of ALD}

Liver biopsy is not necessary for the diagnosis of ALD except when other concomitant diseases are associated like viral disease or NAFLD or diagnosis in doubt due to unreliable history and laboratory findings. Significant history of alcohol intake along with radiological, biochemical and clinical findings are sufficient for diagnosis 
Table 1 Clinical, biochemical and Imaging in alcoholic liver disease

\begin{tabular}{|c|c|c|c|}
\hline Spectrum & Clinical features & Imaging & Laboratory findings \\
\hline \multirow{2}{*}{$\begin{array}{l}\text { Alcoholic } \\
\text { fatty liver }\end{array}$} & \multirow{2}{*}{ Hepatomegaly on examination } & $\begin{array}{l}\text { MRI abdomen: in and out of phase for fat } \\
\text { for detecting hepatic steatosis }\end{array}$ & \multirow{2}{*}{$\begin{array}{l}\text { Normal liver function test or } \\
\text { slightly elevated liver enzymes } \\
\text { (AST, ALT and GGT) and imaging } \\
\text { shows fatty liver }\end{array}$} \\
\hline & & $\begin{array}{l}\text { Transient elastography with CAP is } \\
\text { emerging imaging }\end{array}$ & \\
\hline \multirow{2}{*}{$\begin{array}{l}\text { Alcoholic } \\
\text { hepatitis }\end{array}$} & \multirow{2}{*}{$\begin{array}{l}\text { Combination of these symptoms and signs: } \\
\text { Jaundice, loss of appetite, RUQ pain or } \\
\text { discomfort, ascites, muscle weakness, } \\
\text { confusion, pedal edema, petechial spots and } \\
\text { sometimes fever, hepatomegaly which may be } \\
\text { tender }\end{array}$} & More of clinical diagnosis & Raised serum bilirubin >3 mg\% \\
\hline & & $\begin{array}{l}\text { Ultrasound abdomen/CT abdomen/MRI } \\
\text { shows features of alcoholic fatty liver } \\
\text { and/or cirrhosis with or without ascites }\end{array}$ & $\begin{array}{l}\text { Raised AST/ALT }>3-5 \text { times } \\
\text { ULN with raised GGT }\end{array}$ \\
\hline \multirow{4}{*}{$\begin{array}{l}\text { Cirrhosis } \\
\text { without } \\
\text { alcoholic } \\
\text { hepatitis }\end{array}$} & \multirow{4}{*}{$\begin{array}{l}\text { May be totally asymptomatic in compensated } \\
\text { cirrhosis or anorexia, weight loss, fatigue, } \\
\text { muscle cramps, ascites, impotence, infertility, } \\
\text { loss of pubic hairs, palmer erythema and pedal } \\
\text { edema. Jaundice and ascites may be present in } \\
\text { decompensated disease. Firm liver which may } \\
\text { feel nodular }\end{array}$} & $\begin{array}{l}\text { USG: coarsened echo pattern and } \\
\text { nodularity of liver }\end{array}$ & $\begin{array}{l}\text { Normal to borderline elevated } \\
\text { liver enzymes with low albumin }\end{array}$ \\
\hline & & $\begin{array}{l}\text { CT and MRI abdomen: are highly } \\
\text { predictable for cirrhosis and any } \\
\text { associated liver lesion }\end{array}$ & Normal to high INR \\
\hline & & $\begin{array}{l}\text { Transient elastography to assess liver } \\
\text { stiffness and response to treatment }\end{array}$ & \\
\hline & & & Elevated bilirubin level \\
\hline $\begin{array}{l}\text { Cirrhosis } \\
\text { with } \\
\text { alcoholic } \\
\text { hepatitis }\end{array}$ & $\begin{array}{l}\text { Severe anorexia, weight loss, fatigue, muscle } \\
\text { cramps, ascites, palmer erythema and pedal } \\
\text { edema. Jaundice and moderate to tense } \\
\text { ascites are present in majority of the patients. } \\
\text { Firm liver which is non tender }\end{array}$ & $\begin{array}{l}\text { USG/CT: triple phase/MRI: triple phase } \\
\text { for assessment of liver cirrhosis and liver } \\
\text { SOL }\end{array}$ & $\begin{array}{l}\text { Elevated bilirubin }(>3-5 \mathrm{mg} \%) \\
\text { raised AST/ALT and ratio is }<2 \text {. } \\
\text { High INR with low hemoglobulin, } \\
\text { albumin and platelet count }\end{array}$ \\
\hline
\end{tabular}

RUQ, right upper quadrant; USG, ultrasonography; CT, computed tomography; LAI, liver attenuation index; MRI, magnetic resonance imaging; CAP, controlled attenuation parameter; AST, aspartate aminotransferase; ALT, alanine aminotransferase; GGT, gammaglutamyltranspeptidase; ULN, upper limit of normal; INR, international normalised ration; SOL, space occupying lesions.

of ALD. Van Ness et al. (15) evaluated patients with raised liver enzymes and biopsy was done in them. Clinical findings and history of significant alcohol use have been found to be comparable to liver biopsy for the diagnosis of ALD with sensitivity of $91 \%$ and specificity of $97 \%$ (15).

$\mathrm{AH}$ is categorised in three terminologies. Definite $\mathrm{AH}$ is diagnosed on the basis of compatible clinical diagnosis and liver biopsy confirming the diagnosis; probable $\mathrm{AH}$ is defined as compatible clinical symptoms in absence of any confounding serology for another concomitant disease but without any liver biopsy and possible $\mathrm{AH}$ is defined as clinically suspicious for $\mathrm{AH}$, presence of any confounding factors such as positive serology for virus, ischemic hepatitis, possible drug-induced liver injury, or uncertain alcohol abuse. It is proposed that patients with possible AH should undergo liver biopsy to confirm the diagnosis for any definitive pharmacologic interventions like steroids $(3,16)$.

ALD is a broad spectrum of disease and it can range from totally asymptomatic disease (as fatty liver) to $\mathrm{AH}$ and advanced ALD which includes complications of cirrhosis like jaundice, ascites, variceal bleeding, hepatic encephalopathy, and hepatocellular carcinoma (HCC). Clinical features of ALD will vary according to disease severity in patient and is tabulated in Table 1. Amount and pattern of alcohol intake strongly influences the clinical outcome of the patients. The clinical course of ALD is strongly influenced by alcohol abstinence and total abstinence is recommended at all stages of ALD (17). Even 
patients with decompensated cirrhosis have poor prognosis in those who continue to take alcohol compared to those who abstain from alcohol Most of the patients show frequent overlap of clinical and pathologic findings of ALD.

\section{Hepatic steatosis (HS) (fatty liver)}

Fatty liver is a common finding in two third of heavy alcohol drinkers and it can be assessed radiologically as early as 7 days of initial alcohol consumption of more than 4 standard drinks per day (18). Fatty liver is asymptomatic in majority of patients or presents with mild right upper quadrant discomfort (RUQ). Liver function tests (LFTs) are either normal with normal liver enzymes or mildly increased serum aminotransferase levels and GGT. Liver enzymes (AST and ALT) never rise five times of upper normal value in majority of patients. Ultrasound of the abdomen shows steatosis of various grades (grade 1 to 3 ). Clinical history along with laboratory findings are sufficient to diagnose this condition and liver biopsy is not required in majority of these patients.

Liver biopsy if done shows macro vesicular steatosis predominantly in the centrilobular zone. There will be no significant inflammation or necrosis in fatty liver disease alone (19). However, the presence of steatosis on liver biopsy is no longer considered a benign condition. In a study by Teli et al. (20), patients with a histologic diagnosis of alcoholic fatty liver without $\mathrm{AH}$ were enrolled $(\mathrm{n}=88)$ and analysed retrospectively. Of these patients $18 \%$ progressed to fibrosis or cirrhosis when followed for a median of 10 years. Continued alcohol intake, female gender and histological features of megamitochondria and perivenular fibrosis and mixed macro and micro vesicular steatosis on liver biopsy were associated with development of fibrosis or cirrhosis. Similarly, in a systemic review by Parker et al. (21) mortality was $6 \%$ per year in patients with steatosis and hence steatosis no longer considered as benign condition.

\section{$A H$}

$\mathrm{AH}$ is a severe form of liver injury with features of both hepatocellular injury and cholestasis that results from significant amount of alcohol consumption during a prolonged period of time. One should always keep a high suspicion for $\mathrm{AH}$ in a patient who presented with recent onset or worsening of jaundice in the setting of significant alcohol use in the past 8 weeks. Clinical diagnosis of $\mathrm{AH}$ is defined as rapid development or worsening of jaundice and liver-related complications like serum total bilirubin $>3 \mathrm{mg} / \mathrm{dL}$; ALT and AST elevated $>1.5$ times the upper limit of normal but less than five times the upper limit of normal with the AST/ALT ratio $>1.5$. Patient should be investigated for other concomitant liver disease to confirm the diagnosis of $\mathrm{AH}(3)$.

Symptoms of patients vary according to severity of $\mathrm{AH}$ and vary from mild and nonspecific in some patients to more severe like deep jaundice and hepatic encephalopathy in some patients. The diagnosis of AH can be made with good sensitivity and specificity thorough good reliable history, physical examination, and laboratory results. Liver biopsy is confirmatory if diagnosis is in doubt, but generally not required in day to day management of these patients. However liver biopsy (transjugular liver biopsy) is required in clinical trials or when diagnosis is in doubt.

Patient with mild AH may be asymptomatic or mildly symptomatic. Clinical examination of abdomen shows hepatomegaly and laboratory tests shows increased serum aminotransferase levels with the AST level twice or greater than the ALT level. Severe disease is associated with various grades of malnutrition, jaundice, fever, malaise, tender hepatomegaly with or without hepatic bruit. These patients with severe AH may develop or presents with ascites, hepatic encephalopathy, and acute kidney injury. Features of systemic inflammatory response syndrome (SIRS) are defined as presence of $\geq 2$ of the following: heart rate $>100$ beats per minute, temperature $>38^{\circ} \mathrm{C}$ or $<36^{\circ} \mathrm{C}$, respiratory rate $>12$ breaths per minute, and white blood cell count $>12,000$ or $<4,000 \mathrm{~mm}$ syndrome (SIRS) may be present in these patients even when all relevant test for infections are negative (3).

$\mathrm{AH}$ has got poor short-term survival, need admission and constant monitoring with good nutrition. Many prognostic models have been developed which includes Maddrey's discriminant function [MDF], Model for End-stage Liver Disease (MELD); age, serum bilirubin, INR, creatinine [ABIC], Glasgow Alcoholic Hepatitis Score [GAHS] and Lille's score. These scores predict short-term mortality with varied sensitivity and specificity. Lille's score also guides the physicians to stop steroid therapy if there is no significant benefit after one week of therapy to avoid side effects of it.

MDF score is commonly used by most of the physician due to ease at bedside, robust data in studies and can be calculated easily. MDF score of $>32$ predict short-term mortality of $30 \%$ at 1 month and $40 \%$ within 6 months. Higher the MDF means higher mortality. The GAHS has shown higher accuracy of $81 \%$ to predict 28 -day overall 
survival compared to MDF score at day 1 of presentation. ABIC score classifies patients of $\mathrm{AH}$ in three groups as risk of mortality (based on 3 months) into low risks (0\%), intermediate risk (30\%), and high risk (75\%). A MELD score $>20$ which defines severe AH predicts $20 \%$ mortality in few studies (22-24). In a study by Daswani et al. (25), 183 patients with severe $\mathrm{AH}$ was enrolled and median MELD score of 26 and had a 90 -day mortality of $44 \%$ and outscores other severity scores.

Liver biopsy will show neutrophil predominant inflammatory infiltrate, Mallory hyaline, ballooned hepatocytes and varied degree of fibrosis or cirrhosis as overlap of these pathological findings are common. Perisinusoidal chicken-wire fibrosis and perivenular fibrosis is a distinctive feature of steatohepatitis.

More than half of the individuals admitted with $\mathrm{AH}$ have underlying cirrhosis and have a significantly increased risk of developing cirrhosis. In a study by Park et al. (21) progression of pre-cirrhotic disease to cirrhosis were $1 \%$ in patients with normal histology, $3 \%$ in patients with HS, $10 \%$ in patients with biopsy suggestive of steatohepatitis and $8 \%(3-19 \%)$ in patients with significant fibrosis. Mortality was significantly higher in patients with cirrhosis compared to those with steatosis.

\section{Alcobolic cirrbosis}

Alcohol is most common hepatotoxic agent and excess and continued intake of alcohol consumption increases the risk of developing cirrhosis. Different studies have shown different dosages as the cause of cirrhosis, however there is no clear dose-dependent relationship between alcohol dosage and risk of development of alcoholic cirrhosis as different factors which include genetic predisposition play a significant role (26). Various associated factors like viral hepatitis, obesity and various metabolic factors like diabetes also add to hepatotoxicity of alcohol and hence risk of cirrhosis (7). Patients with cirrhosis and portal hypertension may be completely asymptomatic in compensated cirrhosis or have peripheral signs of chronic liver disease if hepatic decompensation exists like ascites, peripheral edema, asterixis and/or mental confusion, jaundice, malnutrition features like reduced muscle mass and easy fatigability.

Patients with alcoholic cirrhosis may have jaundice as well as tender hepatomegaly if associated $\mathrm{AH}$ is present. These patients may or may not have ascites. If their hepatitis is severe patient will have asterixis and exhibit mental confusion on examination. On examination these patients may have ascites, splenomegaly, parotid enlargement, testicular atrophy, loss of pubic hairs and palmer erythema. In addition, patients with ALD typically have co-morbidities due to the concomitant toxic effects of alcohol on other organ systems and may have signs of peripheral neuropathy, muscle wasting and heart failure.

Liver biopsy is normally not required for the confirmation of alcoholic cirrhosis. However, if there is any doubt, no definitive history of significant alcohol intake in the recent past, associated $\mathrm{AH}$ which require steroid treatment or associated co infection with viral disease, one may need to do transjugular liver biopsy for proper evaluation of aetiology of liver cirrhosis. Alcoholic cirrhosis liver is characterized by small regenerative nodules (micronodular). Absolute abstinence of alcohol significantly improves long-term prognosis and hence should be reinforced in every patient (27).

\section{Summary}

ALD is one of the commonest causes of liver disease. ALD comprises spectrum which include fatty liver, AH, cirrhosis with or without $\mathrm{AH}$ with its complications like ascites, hepatic encephalopathy and HCC. Diagnosis of ALD requires good reliable history of significant alcohol abuse and excluding other causes of liver disease. $\mathrm{AH}$ with alcoholic cirrhosis is one of commonest of acute on chronic liver failure with associated organ failure and high shortterm mortality. These patients present with rapid onset jaundice, ascites, hepatic encephalopathy and acute kidney injury. Liver biopsy is generally not required in majority of patients except when history of alcohol intake is not reliable, associated infection with viral disease, obesity or other hepatotropic or non-hepatotropic insult and treatment protocols under trials when definitive diagnosis of $\mathrm{AH}$ is required.

\section{NAFLD}

NAFLD is a spectrum that ranges from simple steatosis, steatohepatitis with or without fibrosis, cirrhosis and ultimately cirrhosis with HCC. NAFLD is characterised as presence of fat in the liver in the absence of significant alcohol use and other causes of fat in the liver like drugs, lipodystrophy, Wilson disease, parenteral nutrition, starvation and viral disease (28). The definition of significant alcohol consumption that has been used in various $\mathrm{NASH}$ clinical trials is ongoing or recent consumption of 
$>14$ standard drinks/week (14 gm alcohol is one standard drink) per week in women and $>21$ standard drinks/week in men. However according to European Association for the Study of the Liver, diagnosis of NAFLD requires the exclusion of both secondary causes and of a daily alcohol consumption $\geq 30 \mathrm{~g}$ for men and $\geq 20 \mathrm{~g}$ for women. NAFLD is often diagnosed incidentally on imaging and is asymptomatic in majority of the patients. NAFLD is currently considered as hepatic manifestation of metabolic syndrome (28).

\section{Common terms used in spectrum of NAFLD}

NAFLD: whole spectrum of fatty liver disease in persons without significant alcohol consumption and it includes fatty liver to steatohepatitis with or without fibrosis to cirrhosis.

NAFL: diagnosed when liver has $\geq 5 \%$ HS without ballooning of the hepatocytes or fibrosis on liver biopsy. The risk of cirrhosis and liver failure is considered minimal during follow-up.

NASH: diagnosed when liver has $\geq 5 \%$ HS with inflammation and ballooning of hepatocyte which indicate liver injury with or without fibrosis. NASH can progress to advanced fibrosis, cirrhosis and its complication like HCC.

NASH cirrhosis: is diagnosed when cirrhosis is diagnosed in a patient with current or previous histological evidence of steatosis or steatohepatitis.

Majority of patients with NAFLD have only liver steatosis which has benign course with no overall increase in liver related mortality. However, follow-up of fatty liver disease, $10-30 \%$ have the potential to progress to NASH and risk of further progression of liver disease and its complications. Patients with NASH cirrhosis are at risk (3\% per year) of developing HCC and should be screened for HCC as patients with cirrhosis of other etiology (29-31). Multiple long-term studies suggest that patients with NAFLD with advanced fibrosis have $60 \%$ liver-related mortality compared with $9 \%$ in NAFLD without advanced fibrosis $(31,32)$. Only $25-40 \%$ of patients with NASH will develop progressive liver fibrosis during follow up and of this only $20-30 \%$ will develop cirrhosis and its complications. The development of cirrhosis is associated with a poor prognosis as 10 -year mortality rate is $20 \%$ for Child-Pugh A disease. Of these compensated cirrhosis patients $45 \%$ will decompensate within 10 years of diagnosis which further increase the mortality rate (31).

A meta-analysis of patients with suspected NAFLD (based on either radiological imaging or histology) and NASH with or without fibrosis found that patients with NAFLD and NASH were at higher risk of fatal and nonfatal cardiovascular events than those without NAFLD (random effect odds ratio $1.64 ; 95 \% \mathrm{CI}, 1.26-2.13$ ) and (odds ratio 2.58 ; 95\% CI, 1.78-3.75) respectively (33).

\section{Risk factor for fatty liver}

Many risk factors are associated with NAFLD and include obesity, advanced age, male, ethnicity, genetics, composition of the diet like high sucrose and fructose), insulin resistance and recently gut flora dysbiosis and associated inflammation. Many components of metabolic syndrome (insulin resistance and dyslipidemia) are associated with NAFLD and NAFLD is now considered as one of the hepatic manifestations of Metabolic syndrome (34-36).

\section{Spectrum of NAFLD}

\section{Steatosis}

Insulin resistance and its interaction with adipose tissue and associated metabolic syndrome are considered as a key step in the pathogenesis of HS. Insulin resistance leads to dysregulation of many hepatic transcription factors and nuclear receptors, leads to alteration of lipid and glucose metabolism which in turn result in fatty liver. Singh and colleagues (37) enrolled biopsy-proven NAFLD (150 with NAFL and 261 with NASH). Patients with NAFL and no fibrosis at baseline progressed to stage 1 of fibrosis over 14.3 years whereas patients with NASH and no fibrosis at baseline had accelerated rate of progression to stage 1 of fibrosis over 7.1 years.

\section{NASH}

NASH is a histological diagnosis and required liver biopsy for its diagnosis. Presence of steatosis, lobular inflammation and hepatocyte ballooning is the histological feature that distinguishes NASH from simple steatosis. NASH risk factors and its progression to fibrosis and cirrhosis have been evaluated in many studies. NAFL advanced to NASH in $23 \%$ of cases over a period of 3 years based on histological diagnosis and follow up biopsies of 52 patients in a study by Wong et al. (38).

Risk factors which increase the likelihood of NASH in patients with NAFL include obesity, older age, female sex, non-African American race, diabetes mellitus, and hypertension (39). 


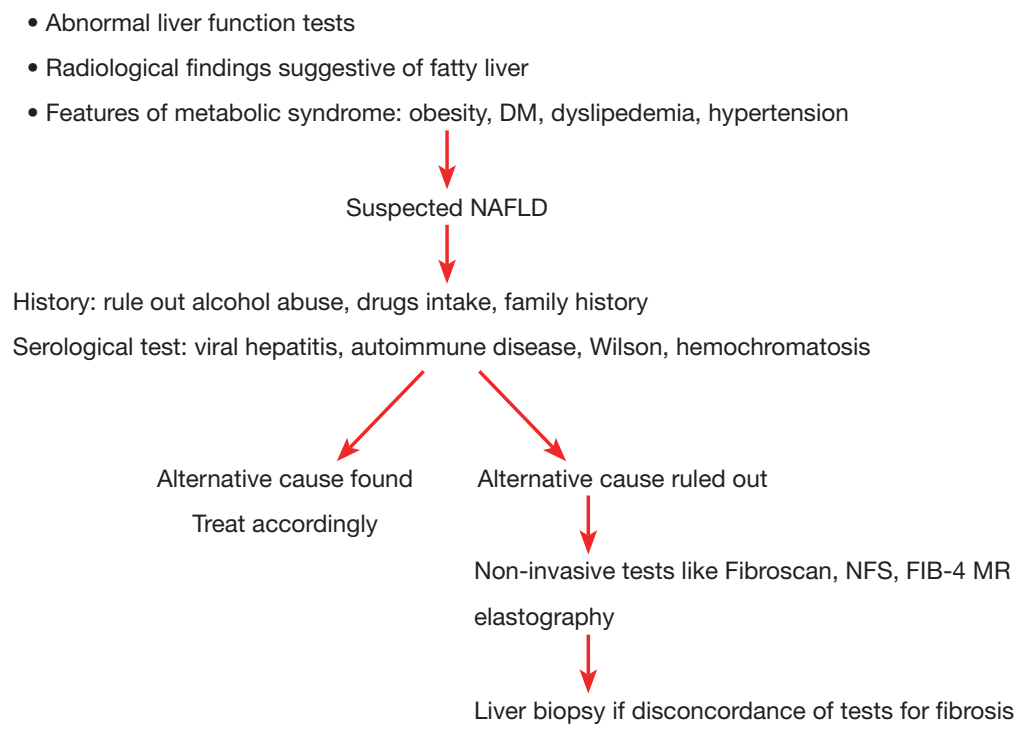

Figure 1 Chart to follow for diagnosis of NAFLD. DM, diabetes mellitus; NAFLD, non-alcoholic fatty liver disease; NFS, NAFLD fibrosis score; FIB-4, fibrosis-4 score; MR, magnetic resonance.

\section{Advanced fibrosis and cirrhosis}

Long-term outcomes studies on natural history of advanced fibrosis and cirrhosis due to NAFLD are limited. In a metaanalysis the global incidence of advanced fibrosis in NASH patients was 67.9 in 1,000 person-years, with $41 \%$ of NASH patients experienced fibrosis progression (average annual progression rate of $0.09 \%)$. NAFLD patients progressed to cirrhosis in $25 \%$ of patients and $7 \%$ to end-stage liver disease (39). Study comparing patient of chronic liver disease due to viral hepatitis patients and HCC, patients with HCC due to NASH related cirrhosis tend to be older and are likely to be females (40). HCC in NAFLD patients has been associated with increasing age, obesity, diabetes, the PNPLA3 I148 M polymorphism (39).

\section{Diagnosis and staging of NAFLD/NASH}

\section{Clinical features}

NAFLD is diagnosed based on clinical history, laboratory and radiological tests and confirmed on histological biopsy findings. However, in clinical practise abdominal imaging which reveals HS is sufficient for diagnosis of NAFLD if other diseases have been rule out. Most of the patient and physician have apprehension about liver biopsy for the diagnosis of NAFL and may not be required in each case after relevant blood tests. However liver biopsy is needed in differentiating NASH from simple steatosis as NASH is a diagnosis based on histology (Figure 1). Diagnosis of
NASH is important as NASH has a higher risk of disease progression as compared to simple steatosis and hence helps in treatment plan and prognostication of disease to the patient (39).

Most of patients with NAFLD are asymptomatic or have non-specific symptoms before the diagnosis is made. Fatigue and right upper abdominal discomfort are common presenting symptoms. Other symptom includes abdominal bloating, irregular bowel habits and decreased sleep. NAFLD is incidentally diagnosed in majority of the patients with abnormal liver enzymes or abnormalities on imaging performed for other reasons or are identified based on clinical features of the metabolic syndrome $(41,42)$. Early diagnosis is often made by physician or hepatologist based on high index of suspicion in patients with metabolic syndrome or subtle elevations of liver enzymes. However liver enzymes may not be raised in many patients irrespective of severity of liver disease. People who develop decompensation of liver disease due to NAFLD may have symptoms of jaundice, ascites, variceal bleed or hepatic encephalopathy.

As NAFLD is considered as hepatic counter part of metabolic syndrome and. It has been seen in imaging that $70-80 \%$ of subjects with central obesity and $50-80 \%$ of patients with type 2 diabetes $(43,44)$. NAFLD has also been reported in patients with dyslipidemia, obstructive sleep apnea syndrome, polycystic ovarian disease, hypopituitarism, vitamin D deficiency, hypopituitarism, 
hypothyroidism and hyperuricemia. These factors should also be assessed simultaneously while ordering the blood tests for the evaluation of NAFLD $(45,46)$.

Most of the patients with NAFLD has normal or near normal LFTs. Patients may show mildly raised transaminases (ALT > AST) and/or GGT and even if elevated, the ALT typically falls (and AST may rise) as fibrosis progresses to cirrhosis. ALT level correlation with histological findings has poor sensitivity and specificity for the diagnosis of NASH $(47,48)$.

Patients with abnormal LFTs, should be simultaneously evaluated for alcohol abuse, drug-induced liver injury, viral hepatitis, autoimmune liver disease, haemochromatosis, celiac disease and Wilson's disease (in patients $<45$ years old). Autoantibodies may be detected in low titre in subjects with NAFLD [antinuclear antibody (ANA) $<1: 100$ and/ or anti-smooth muscle antibody (ASMA) <1:40] but these patients are associated with normal IgG levels. Few patients may have high ferritin levels and usually reflect underlying inflammatory activity or insulin resistance (49). Liver biopsy should be considered if there is any doubt in diagnosis.

Scoring symptoms: NAFLD is common symptomatic problem and liver biopsy for the diagnosis and follows up after treatment is not a preferred choice by many patients. To overcome this problem non-invasive scoring systems for the assessment and treatment follow up of fibrosis in NAFLD have been evaluated in many studies. Transient elastography, fibrosis-4 score (FIB-4), NAFLD fibrosis score (NFS), BARD (BMIAST/ALT-Diabetes), enhanced liver fibrosis panel (ELF), Fibro Meter, Fibro Test are few of the commonly used non-invasive scoring system. Of these scoring system NFS and FIB-4 have shown good sensitivity and specificity compared to liver biopsy in predicting advanced fibrosis in patients with NAFLD. These non-invasive scoring system like NFS and FIB4 have been recommended by American Association for the Study of Liver Diseases (AASLD) in the diagnosis of fibrosis for NAFLD patients (28,50-53).

\section{Summary}

NAFLD is characterised by presence of hepatic fat accumulation after the exclusion of other causes of HS like hepatitis B and C, excessive alcohol consumption, drugs and parenteral nutrition and prolonged starvation. NAFLD clinical spectrum ranges from NAFL to NASH, advanced fibrosis, cirrhosis, and finally HCC in some patients. NAFLD is increasing all over the world so the
NASH related cirrhosis and its complications. NASH related cirrhosis has become a common indication for liver transplantation. Most of the patients with NAFLD have associated metabolic comorbidities like obesity, type II diabetes, dyslipidemia, and metabolic syndrome. NAFLD patients are asymptomatic in majority of cases and are diagnosed on tests done for some other reason. Few patients with NAFLD may present with fatigue, dyspepsia, dull pain in right upper abdomen and hepatosplenomegaly. Morbidity and mortality in patients with NALFD correlate with fibrosis stage. In view of invasive nature of liver biopsy and reluctance from the patients to undergo repeated such procedure, non-invasive methods are commonly used. Noninvasive scoring systems are helpful at extremes of fibrosis but have poor sensitivity and specificity in diagnosis less advanced fibrosis. Liver biopsy is usually done for such cases to accurately determine the degree of fibrosis.

\section{Acknowledgments}

None.

\section{Footnote}

Conflicts of Interest: The authors have no conflicts of interest to declare.

Ethical Statement: The authors are accountable for all aspects of the work in ensuring that questions related to the accuracy or integrity of any part of the work are appropriately investigated and resolved.

\section{References}

1. Mandayam S, Jamal MM, Morgan T. Epidemiology of alcoholic liver disease. Semin Liver Dis 2004;24:217-32.

2. Estes C, Razavi H, Loomba R, et al. Modeling the epidemic of nonalcoholic fatty liver disease demonstrates an exponential increase in burden of disease. Hepatology 2018;67:123-33.

3. Singal AK, Bataller R, Ahn J, et al. ACG Clinical Guideline: Alcoholic Liver Disease. Am J Gastroenterol 2018;113:175-94.

4. Chalasani N, Younossi Z, Lavine JE, et al. The diagnosis and management of nonalcoholic fatty liver disease: Practice guidance from the American Association for the Study of Liver Diseases. Hepatology 2018;67:328-57.

5. Saunders JB, Aasland OG, Babor TF, et al. Development 
of the alcohol use disorders identification test (AUDIT): WHO Collaborative Project on Early Detection of Persons with Harmful Alcohol Consumption--II. Addiction 1993;88:791-804.

6. Bradley KA, DeBenedetti AF, Volk RJ, et al. AUDIT-C as a brief screen for alcohol misuse in primary care. Alcohol Clin Exp Res 2007;31:1208-17.

7. Hart CL, Morrison DS, Batty GD et al. Effect of body mass index and alcohol consumption on liver disease: analysis of data from two prospective cohort studies. BMJ 2010;340:c1240.

8. Singal AK, Kuo YF, Anand BS. Hepatitis C virus infection in alcoholic hepatitis: prevalence patterns and impact on in-hospital mortality. Eur J Gastroenterol Hepatol 2012;24:1178-84.

9. O'Shea RS, Dasarathy S, McCullough AJ. Practice Guideline Committee of the American Association for the Study of Liver Diseases, Practice Parameters Committee of the American College of Gastroenterology. Alcoholic liver disease. Hepatology 2010;51:307-28.

10. Bell H, Skinningsrud A, Raknerud N, et al. Serum ferritin and transferrin saturation in patients with chronic alcoholic and non-alcoholic liver diseases. J Intern Med 1994;236:315-22.

11. Litten RZ, Bradley AM, Moss HB. Alcohol biomarkers in applied settings: recent advances and future research opportunities. Alcohol Clin Exp Res 2010;34:955-67.

12. Staufer K, Andresen H, Vettorazzi E, et al. Urinary ethyl glucuronide as a novel screening tool in patients pre- and post-liver transplantation improves detection of alcohol consumption. Hepatology 2011;54:1640-9.

13. Deas D, Johnson N, Thomas S. Carbohydrate deficient transferrin (CDT) predicts heavy drinking in adolescents with alcohol dependence. Alcohol 2019;81:27-30.

14. Hock B, Schwarz M, Domke I, et al. Validity of carbohydrate-deficient transferrin (\%CDT), gammaglutamyltransferase (gamma-GT) and mean corpuscular erythrocyte volume (MCV) as biomarkers for chronic alcohol abuse: a study in patients with alcohol dependence and liver disorders of non-alcoholic and alcoholic origin. Addiction 2005;100:1477-86.

15. Van Ness MM, Diehl AM. Is liver biopsy useful in the evaluation of patients with chronically elevated liver enzymes? Ann Intern Med 1989;111:473-8.

16. Crabb DW, Bataller R, Chalasani NP et al. Standard definitions and common data elements for clinical trials in patients with alcoholic hepatitis: recommendation from the NIAAA Alcoholic Hepatitis Consortia. Gastroenterology
2016;150:785-90.

17. Verrill C, Markham H, Templeton A, et al. Alcohol-related cirrhosis-early abstinence is a key factor in prognosis, even in the most severe cases. Addiction 2009;104:768-74.

18. Fleming KA, McGee JO. Alcohol induced liver disease. J Clin Pathol 1984;37:721-33.

19. Savolainen V, Perola M, Lalu K, et al. Early perivenular fibrogenesis-precirrhotic lesions among moderate alcohol consumers and chronic alcoholics. J Hepatol 1995;23:524-31.

20. Teli MR, Day CP, Burt AD, et al. Determinants of progression to cirrhosis or fibrosis in pure alcoholic fatty liver. Lancet 1995;346:987-90.

21. Parker R, Aithal GP, Becker U, et al. Natural history of histologically proven alcohol-related liver disease: A systematic review. J Hepatol 2019;71:586-93.

22. Maddrey WC, Boitnott JK, Bedine MS, et al. Corticosteroid therapy of alcoholic hepatitis. Gastroenterology 1978;75:193-9.

23. Forrest EH, Evans CD, Stewart S, et al. Analysis of factors predictive of mortality in alcoholic hepatitis and derivation and validation of the Glasgow alcoholic hepatitis score. Gut 2005;54:1174-9.

24. Louvet A, Naveau S, Abdelnour M, et al. The Lille model: a new tool for therapeutic strategy in patients with severe alcoholic hepatitis treated with steroids. Hepatology 2007;45:1348-54.

25. Daswani R, Kumar A, Anikhindi SA, et al. Predictors of 90-day mortality in patients with severe alcoholic hepatitis: Experience with 183 patients at a tertiary care center from India. Indian J Gastroenterol 2018;37:141-52 .

26. Corrao G, Bagnardi V, Zambon A, et al. Meta-analysis of alcohol intake in relation to risk of liver cirrhosis. Alcohol Alcohol 1998;33:381-92.

27. Pessione F, Ramond MJ, Peters L, et al. Five-year survival predictive factors in patients with excessive alcohol intake and cirrhosis. Effect of alcoholic hepatitis, smoking and abstinence. Liver Int 2003;23:45-53.

28. Chalasani N, Younossi Z, Lavine J, et al. The diagnosis and management of non-alcoholic fatty liver disease: practice guideline by the American Association for the Study of Liver Diseases, American College of Gastroenterology, and the American Gastroenterological Association. Hepatology 2012;5 5:2005-23.

29. Matteoni CA, Younossi ZM, Gramlich T, et al. Non alcoholic fatty liver disease: a spectrum of clinical and pathological severity. Gastroenterology 1999;116:1413-9

30. Dam-Larsen S, Franzmann M, Andersen IB, et al. Long 
term prognosis of fatty liver: risk of chronic liver disease and death. Gut 2004;53:750-5.

31. Ekstedt M, Franzen LE, Mathiesen UL, et al. Long-term follow-up of patients with NAFLD and elevated liver enzymes. Hepatology 2006;44:865-73.

32. Angulo P. The natural history of NAFLD. In: Farrell GC, McCullough AJ, Day CP. editors. Non-alcoholic fatty liver disease: a practical guide. London: Wiley Blackwell Press, 2013:37-45.

33. Targher G, Byrne CD, Lonardo A, et al. Non-alcoholic fatty liver disease and risk of incident cardiovascular disease: a meta-analysis. J Hepatol 2016;65:589-600.

34. Musso G, Gambino R, De Michieli F, et al. Dietary habits and their relations to insulin resistance and postprandial lipemia in nonalcoholic steatohepatitis. Hepatology 2003;37:909-16.

35. Ouyang X, Cirillo P, Sautin Y, et al. Fructose consumption as a risk factor for non-alcoholic fatty liver disease. J Hepatol 2008;48:993-9.

36. Cortez-Pinto H, Jesus L, Barros H, et al. How different is the dietary pattern in non-alcoholic steatohepatitis patients? Clin Nutr 2006;25:816-23.

37. Singh S, Allen AM, Wang Z, et al. Fibrosis progression in nonalcoholic fatty liver vs nonalcoholic steatohepatitis: a systematic review and meta-analysis of paired-biopsy studies. Clin Gastroenterol Hepatol 2015;13:643-54.e1-9; quiz e39-40.

38. Wong VW, Wong GL, Choi PC, et al. Disease progression of nonalcoholic fatty liver disease: a prospective study with paired liver biopsies at 3 years. Gut 2010;59: 969-74.

39. Younossi ZM, Koenig AB, Abdelatif D, et al. Global epidemiology of non-alcoholic fatty liver diseaseMeta-analytic assessment of prevalence, incidence, and outcomes. Hepatology 2016;64:73-84.

40. Bugianesi E, Leone N, Vanni E, et al. Expanding the natural history of non-alcoholic steatohepatitis: from cryptogenic cirrhosis to hepatocellular carcinoma. Gastroenterology 2002;123:134-40.

41. Leite NC, Salles G, Araujo A, et al. Prevalence and associated factors of non-alcoholic fatty liver disease in patients with type-2 diabetes mellitus. Liver Int 2009;29:113-9.

42. Williams CD, Stengel J, Asike M, et al. Prevalence of nonalcoholic fatty liver disease and nonalcoholic steatohepatitis among a largely middle-aged population utilizing ultrasound and liver biopsy: a prospective study. Gastroenterology 2011;140:124-31.

43. Jimba S, Nakagami T, Takahashi M, et al. Prevalence of non-alcoholic fatty liver disease and its association with impaired glucose metabolism in Japanese adults. Diabet Med 2005;22:1141-5.

44. Williamson RM, Price JF, Glancy S, et al. Prevalence of and risk factors for hepatic steatosis and nonalcoholic Fatty liver disease in people with type 2 diabetes: the Edinburgh Type 2 Diabetes Study. Diabetes Care 2011;34:1139-44.

45. Gambarin-Gelwan M, Kinkhabwala S, Schiano T, et al. Prevalence of non-alcoholic fatty liver disease in women with polycystic ovary syndrome. Clin Gastroenterol Hepatol 2007;5:496-501.

46. Chin K, Nakamura T, Takahashi K, et al. Effects of obstructive sleep apnea syndrome on serum aminotransferase levels in obese patients. Am J Med 2003;114:370-6.

47. Mandal A, Bhattarai B, Kafle P, et al. Elevated Liver Enzymes in Patients with Type 2 Diabetes Mellitus and Non-alcoholic Fatty Liver Disease. Cureus 2018;10:e3626.

48. Mofrad P, Contos MJ, Haque M, et al.: Clinical and histologic spectrum of nonalcoholic fatty liver disease associated with normal ALT values. Hepatology 2003, 37:1286-92.

49. Kowdley KV, Belt P, Wilson LA, et al. Serum ferritin is an independent predictor of histologic severity and advanced fibrosis in patients with nonalcoholic fatty liver disease. Hepatology 2012;55:77-85.

50. Shah AG, Lydecker A, Murray K, et al. Comparison of noninvasive markers of fibrosis in patients with nonalcoholic fatty liver disease. Clin Gastroenterol Hepatol 2009; 7:1104-12.

51. Machado MV, Cortez-Pinto H. Non-invasive diagnosis of non-alcoholic fatty liver disease. A critical appraisal. J Hepatol 2013;58:1007-19.

52. Wong VW, Vergniol J, Wong GL, et al. Diagnosis of fibrosis and cirrhosis using liver stiffness measurement in non-alcoholic fatty liver disease. Hepatology 2010;51:454-62.

53. Guha IN, Parkes J, Roderick P, et al. Noninvasive markers of fibrosis in nonalcoholic fatty liver disease: Validating the European Liver Fibrosis Panel and exploring simple markers. Hepatology 2008;47:455-60.

doi: $10.21037 / \operatorname{tgh} .2019 .10 .02$

Cite this article as: Sharma $\mathrm{P}$, Arora A. Clinical presentation of alcoholic liver disease and non-alcoholic fatty liver disease: spectrum and diagnosis. Transl Gastroenterol Hepatol 2020;5:19. 